Original Research Article

\title{
Nephroprotective effect of turmeric on oxidative stress, renal histopathology and toxicity induced by gentamicin
}

\author{
Sangita Devrao Jogdand ${ }^{1 *}$, Raju Shinde ${ }^{2}$, Vivek Sinha ${ }^{2}$, Naman Chandrakar ${ }^{2}$
}

\author{
${ }^{1}$ Department of Pharmacology, \\ Jawaharlal Nehru Medical \\ College, Sawangi Meghe \\ Wardha, Maharashtra, India \\ ${ }^{2}$ Department of Surgery, \\ Jawaharlal Nehru Medical \\ College, Sawangi Meghe \\ Wardha, Maharashtra, India
}

Received: 24 April 2017 Accepted: 27 April 2017

\section{*Correspondence to:}

Dr. Sangita Devrao Jogdand, Email: drsangitaraj@gamil.com

Copyright: (C) the author(s), publisher and licensee Medip Academy. This is an openaccess article distributed under the terms of the Creative Commons Attribution NonCommercial License, which permits unrestricted noncommercial use, distribution, and reproduction in any medium, provided the original work is properly cited.

\begin{abstract}
Background: Commonly used aminoglycosides have frequent side effect of nephrotoxicity, still are preferred by clinicians because of efficacy against gram negative bacteria, resistant bacteria, nosocomial infections and cost effectiveness. Gentamicin produces oxidative stress; substances ameliorating stress are used to reduce toxicity. Turmeric has multiple medicinal properties including potent antioxidant activity, hence study was undertaken.

Methods: Eight groups containing six animals in each group, treated for 15 days. First group treated with normal saline. Second, fourth and sixth group treated with only gentamicin- sacrificed at $16,22,29^{\text {th }}$ day. Third, fifth and seventh group treated with gentamicin and turmeric simultaneously and sacrificed on 16, 22, $29^{\text {th }}$ day. Eighth group was pre-treated with turmeric for thirty days and concurrently treated with gentamicin and turmeric for 15 days and sacrificed on 16th day. Levels of blood urea, serum creatinine, superoxide dismutase and histopathological grades were assessed each time.

Results: Severe renal dysfunction $(146 \pm 9.2,2.03 \pm 0.26)$, highest renal injury grading $(3.66 \pm 0.24)$ was observed in only gentamicin treated groups followed by spontaneous recovery after withdrawal of drug but with higher levels of oxidative stress $(0.04 \pm 0.01)$. Gentamicin and Turmeric treated groups maintained renal function and had lower level of renal damage grades and oxidative stress. Turmeric pre-treated group was having lowest oxidative stress $(0.12 \pm 0.03)$, histopathology grade $(0.60 \pm 0.06)$ with normal renal functions.

Conclusions: Turmeric has potent antioxidant property which effectively protects kidney from damage induced because of gentamicin.
\end{abstract}

Keywords: Gentamicin, Nephrotoxicity, Oxidative stress, Turmeric

\section{INTRODUCTION}

Aminoglycoside antibiotics are widely used as first or second choice antibiotics in the treatment of complex gram negative infections like, urinary, intestinal and pulmonary due high stability of drug and cost effectiveness.

This group is known for their ototoxicity and nephrotoxicity leading to renal impairment in about 10 to 25 percent of the patients. Increased toxicity is observed if other risk factors are present; like hypothyroidism, hepatic dysfunction, reduced renal mass and concomitant use of other commonly used nephrotoxic drugs like NSAIDs, diuretics and cephalosporins. It has confirmed by both in vitro and in vivo studies that gentamicin produce oxidative stress which is key reason for its toxicity. ${ }^{1,2}$

Toxicity of gentamicin is related to its preferential accumulation in proximal convoluted tubules, pathologically characterised by granulovacuolar debris, epithelial necrosis, desquamation and clinically manifested as azotemia. ${ }^{2,3}$

Various chemical compounds and reactions produce toxic oxygen species called as pro-oxidants or free radicals. Free radicals may be oxygen derived like superoxide $\left(\mathrm{O}_{2}\right)$, Hydroxyl (HO-), hydrogen peroxide $\left(\mathrm{H}_{2} \mathrm{O}_{2}\right)$ or nitrogen derived like Nitric oxide (NO), peroxinitrite (ONOO-). 
Electromagnetic radiation, cigarette smoke, UV light are exogenous sources while mitochondrial electron transport chain, beta oxidation of fat, auto oxidation of catecholamines are endogenous sources. Excess production of pro-oxidants cause damage to cell. Normally in cell there is a balance between pro-oxidants and antioxidants. Excess reactive oxygen species (ROS) cause increased peroxidation of membrane phospholipids, oxidative modification of proteins and mitochondrial DNA damage leads cell aging and toxicity. ${ }^{4}$

Antioxidants are the substance when present in low quantity significantly delays or prevents oxidation of substrate like lipids, proteins, carbohydrates and DNA. They may be enzymatic, non-enzymatic or plant derived. Enzymatic antioxidants are superoxide dismutase (SOD) which catalyse breakdown of superoxide, catalase (CAT) involved in reduction of $\mathrm{H}_{2} \mathrm{O}_{2}$. Non-enzymatic antioxidants include Vitamin-E, Vitamin-C. Plant derived antioxidants include polyphenol, phenolic acid, caretenoids. Curcumin- a bioactive ingredient of turmeric is having multiple properties like antiseptic, antiinflammatory, analgesic, anti-cancerous and most importantly antioxidant activity. It acts as scavenger for ROS, as well it protects haemoglobin from oxidation. Study supported that antioxidant property reduces the toxicity of lead by significant increase in superoxide dismutase, reduced glutathione and catalase activities. Hence the present study was undertaken to study the effect of turmeric on oxidative stress and nephrotoxicity induced by commonly used drug gentamicin. ${ }^{5,6}$

Aim of the study was to evaluate antioxidant property of turmeric and its protective effect on renal histopathology and nephrotoxicity induced by gentamicin in albino rats.

Objectives of the study were a) to study oxidative stress due to gentamicin. b) to study histopathological grade of renal injury. c) to study spontaneous recovery of kidney function in gentamicin toxicity. d) to study short term and long term effect of turmeric treatment on gentamicin toxicity.

\section{METHODS}

Study was conducted at SRTR medical college Ambajogai. Healthy albino rats of weights between 150200 gms were used for the study.

Animals were kept on standard pellet diet after acclimatization to environment. Animals were divided in to eight groups having six animals in each group. Nephrotoxicity was induced by injecting gentamicin Intraperitoneally (IP) at dose of $80 \mathrm{mg} / \mathrm{kg} /$ day for 15 days.

Turmeric was collected locally and powder was prepared. Suspension of $3 \%$ was prepared in gum acasia. A calculated dose of test drug $200 \mathrm{mg} / \mathrm{kg} /$ day per orally (PO) and GM was given as per protocol of the groups.

\section{Study design}

\section{Control group}

Group I: Received normal saline $2 \mathrm{ml}$ IP for 15 days. Animals were sacrificed on 16th day for biochemical investigations and histopathological examinations.

\section{Gentamicin toxicity}

Group II: This group received only gentamicin $80 \mathrm{mg} / \mathrm{kg} / \mathrm{d}$ IP for 15 days. Animals were sacrificed on 16th day for biochemical investigations and histopathological examinations.

\section{Concurrent GM and turmeric}

Group III: This group received gentamicin $80 \mathrm{mg} / \mathrm{kg} / \mathrm{d}$ IP and turmeric $200 \mathrm{mg} / \mathrm{kg} / \mathrm{d} / \mathrm{PO}$ for 15 days. Animals were sacrificed on 16th day for biochemical investigations and histopathological examinations.

\section{GM recovery}

Group IV: This group received gentamicin $80 \mathrm{mg} / \mathrm{kg} / \mathrm{d}$ IP for 15. Animals were sacrificed on $22^{\text {nd }}$ day for biochemical investigations and histopathological examinations.

\section{Concurrent GM and turmeric recovery}

Group V: This group received gentamicin $80 \mathrm{mg} / \mathrm{kg} / \mathrm{d}$ IP and turmeric $200 \mathrm{mg} / \mathrm{kg} / \mathrm{d} / \mathrm{PO}$ for 15 days. Animals were sacrificed on $22^{\text {nd }}$ day for biochemical investigations and histopathological examinations.

\section{GM recovery}

Group VI: This group received gentamicin $80 \mathrm{mg} / \mathrm{kg} / \mathrm{d}$ IP for 15. Animals were sacrificed on $29^{\text {th }}$ day for biochemical investigations and histopathological examinations.

\section{Concurrent GM and turmeric recovery}

Group VII: This group received gentamicin $80 \mathrm{mg} / \mathrm{kg} / \mathrm{d}$ IP and turmeric $200 \mathrm{mg} / \mathrm{kg} / \mathrm{d} / \mathrm{PO}$ for 15 days. Animals were sacrificed on $29^{\text {th }}$ day for biochemical investigations and histopathological examinations.

Long term Turmeric Group VIII- This group received turmeric $200 \mathrm{mg} / \mathrm{kg} / \mathrm{day} / \mathrm{PO}$ for 30 days followed by turmeric in same dose and gentamicin $80 \mathrm{mg} / \mathrm{kg} /$ day IP for 15 day. Animals were sacrificed on 46 days for assessment of renal function and histopathological examination. In the study, all animals were sacrificed under ether anaesthesia. Blood collected from heart by cardiac puncture, both kidneys were dissected out from retroperitoneum. 


\section{Assessment of renal toxicity}

Renal dysfunction was assessed by estimation of blood urea levels by DAM method, serum creatinine levels by picric acid method and Histopathological grade of renal damage assessed and highest grade of renal damage was considered for statistical comparisons.

Protective effect of Turmeric was assessed by estimation of superoxide dismutase (SOD).

\section{Histopathological grades of renal damage}

Both kidneys from all animals were removed, processed and embedded in paraffin wax. Sections stained with Haematoxylin and Eosin ( $\mathrm{H}$ and $\mathrm{E}$ ) were examined under light microscope and score was given as per damage as follows.

- Score-0: Normal

- Score-1: Areas of focal granulovacuolar debris with or without evidence of tubular epithelial cell desquamation of small foci. $(<1 \%$ of total tubular population)

- Score-2: Tubular epithelial necrosis and desquamation easily seen but involving less than half of cortical tubules

- Score-3: More than half of proximal tubules showing desquamation and necrosis but involved tubules easily found

- Score-4: Complete or almost complete tubular necrosis. ${ }^{7}$

\section{Statistical analysis}

All values were expressed as mean with SD. For estimation of total variation present in a set of data, all groups were first subjected to ANOVA for measurement of significance between two groups, the student's t test was used and $\mathrm{p}$ Value $<0.05$ was considered significant.

\section{RESULTS}

Gentamicin at a dose $80 \mathrm{mg} / \mathrm{kg}$ for 15 day had induced severe renal dysfunction, depletion of superoxide dismutase and marked damage to renal parenchyma (3.66 $\pm 0.24)$. There was spontaneous recovery in renal function to normal in all animals within one week duration after withdrawal i.e. at $21^{\text {st }}$ day and maintained thereafter i.e. at $28^{\text {th }}$ day. These results suggest that nephrotoxicity due to gentamicin is transient and reversible after drugs has been discontinued. Oxidative stress was decreased after withdrawal of gentamicin which hallmarked by increased levels of SOD at $21^{\text {st }}$ and $28^{\text {th }}$ day compared to $15^{\text {th }}$ day (Table 1, Table 2).

Table 1: Group 1 normal saline treated group (15 days).

\begin{tabular}{|l|l|}
\hline Parameters & Mean with SD \\
\hline Blood urea & $20.68 \pm 1.35$ \\
\hline Serum creatinine & $0.99 \pm 0.06$ \\
\hline Superoxide dismutase & $0.31 \pm 0.01$ \\
\hline Renal histopathological grade & - \\
\hline
\end{tabular}

In the groups treated with turmeric and gentamicin simultaneously, all animals maintained normal renal functions at $15^{\text {th }}, 21^{\text {st }}$, and $28^{\text {th }}$ day, though there was renal parenchymal damage $(1.98 \pm 0.07)$, grades of renal parenchymal damage were less than that of only gentamicin treated groups to extent that these groups maintained normal renal functions and there was higher serum concentration of SOD at all the phases of assessment i.e. at the $16^{\text {th }}, 22^{\text {nd }}$, and $29^{\text {th }}$ days of assessment (Table 3).

Table 2: Gentamicin treated groups.

\begin{tabular}{|llll|}
\hline Parameters & $\begin{array}{l}\text { Gentamicin treated group-2 } \\
\text { (after 15 days) } \\
\text { Mean with SD }\end{array}$ & $\begin{array}{l}\text { Gentamicin treated group-4 } \\
\text { (after 21 days) } \\
\text { Mean with SD }\end{array}$ & $\begin{array}{l}\text { Gentamicin treated } \\
\text { group-6 (after 28 days) } \\
\text { Mean with SD }\end{array}$ \\
\hline $\begin{array}{l}\text { Blood urea } \\
\text { Serum creatinine }\end{array}$ & $2.03 \pm 0.26$ & $24.17 \pm 2.63$ & $23.92 \pm 2.23$ \\
\hline Superoxide dismutase & $0.04 \pm 0.01$ & $0.99 \pm 0.11$ & $0.99 \pm 0.13$ \\
\hline $\begin{array}{l}\text { Renal histopathological } \\
\text { grade }\end{array}$ & $3.66 \pm 0.24$ & $0.05 \pm 0.01$ & $0.05 \pm 0.01$ \\
\hline
\end{tabular}

Table 3: Gentamicin+turmeric treated groups.

\begin{tabular}{|llll|} 
Parameters & $\begin{array}{l}\text { Gentamicin+turmeric } \\
\text { treated group-3 } \\
\text { (after 15 days) } \\
\text { Mean with SD }\end{array}$ & $\begin{array}{l}\text { Gentamicin+turmeric } \\
\text { treated group-5 } \\
\text { (after 21 days) } \\
\text { Mean with SD }\end{array}$ & $\begin{array}{l}\text { Gentamicin+turmeric } \\
\text { treated group-7 } \\
\text { (after 28 days) } \\
\text { Mean with SD }\end{array}$ \\
\hline Blood urea & $27.68 \pm 4.33$ & $23.94 \pm 1.35$ & $26.56 \pm 2.16$ \\
\hline Serum creatinine & $0.87 \pm 0.07$ & $0.86 \pm 0.05$ & $0.44 \pm 0.09$ \\
\hline Superoxide dismutase & $0.11 \pm 0.06$ & $0.09 \pm 0.01$ & $0.09 \pm 0.01$ \\
\hline Renal histopathological grade & $1.98 \pm 0.07$ & $1.18 \pm 0.09$ & $0.86 \pm 0.16$ \\
\hline
\end{tabular}


The group which received long term treatment with turmeric for thirty days prior to administration of gentamicin and turmeric for 15 days, all animals maintained normal kidney functions and grade of renal parenchymal damage of lowest in this group $(0.60 \pm 0.06)$. When compared with short term treated group of turmeric it showed that there were highest levels of super oxide dismutase. $(0.12 \pm 0.03 \mathrm{~V} / \mathrm{S} 0.11 \pm 0.06)$ (Table 4). All the parameters except blood urea level were at statistical significant difference in gentamicin and turmeric treated group (Table 5).
Table 4: Group 8 pre-treatment with turmeric for 30 days followed by gentamicin and turmeric treatment for $\mathbf{1 5}$ days.

\begin{tabular}{|l|l|}
\hline Parameters & Mean with SD \\
\hline Blood urea & $21.15 \pm 2.50$ \\
\hline Serum creatinine & $0.85 \pm 0.13$ \\
\hline Superoxide dismutase & $0.12 \pm 0.03$ \\
\hline Renal histopathological grade & $0.60 \pm 0.06$ \\
\hline
\end{tabular}

Table 5: Comparisons of means and SD of different groups.

\begin{tabular}{|lllllll|}
\hline Parameters & $\begin{array}{l}\text { Group-1 } \\
\text { V/s group-2 }\end{array}$ & $\begin{array}{l}\text { Group-2 } \\
\text { V/s group-3 }\end{array}$ & $\begin{array}{l}\text { Group-1 } \\
\text { V/s group-3 }\end{array}$ & $\begin{array}{l}\text { Group-4 V/s } \\
\text { group-5 }\end{array}$ & $\begin{array}{l}\text { Group-6 V/s } \\
\text { group-7 }\end{array}$ & $\begin{array}{l}\text { Group-1 V/s } \\
\text { group-8 }\end{array}$ \\
\hline Blood urea & 0.0001 & 0.0001 & 0.0032 & 0.85 & 0.0639 & 0.69 \\
\hline Serum creatinine & 0.0001 & 0.0001 & 0.0097 & 0.02 & 0.0001 & 0.03 \\
\hline $\begin{array}{l}\text { Superoxide dismutase } \\
\begin{array}{l}\text { Renal histopathological } \\
\text { grade }\end{array}\end{array}$ & 0.0001 & 0.0001 & 0.0094 & 0.0001 & 0.0001 & 0.0001 \\
\hline
\end{tabular}

\section{DISCUSSION}

The present study was conducted in the albino rat to explore the nephroprotective effect of turmeric on the biochemical changes in the renal function, oxidative stress and extent of histopathological changes in the renal parenchyma after administration of gentamicin.

Serum level of super oxide dismutase (SOD) was used to determine increase in the defence mechanism as oxidative stress induced by gentamicin markedly reduces levels of SOD. It was observed in the present study that gentamicin at a dose $80 \mathrm{mg} / \mathrm{kg}$ for 15 days had induced severe renal dysfunction. Functional derangements are multifactorial and mainly due to:

- Preferential accumulation to proximal convoluted tubules

- Induction of inflammatory response

- Production of ROS

- Decreased renal blood flow

- Derangements of antioxidant due to its ability to alter mitochondrial respiration.

Gentamicin is transferred through megalin ligands by endocytosis which then transferred to lysosome, and by ultimately binding to acid phospholipids ending into production of phospholipids metabolites. ${ }^{8}$

It is observed that toxicity of the gentamicin is dose dependent and increasing dose produces more severe dysfunctions, concentration of gentamicin can as high as 30 folds in proximal convoluted tubules than plasma concentration and continuous infusion is more toxic than intermittent dosing, hence in clinical practices lower dose or frequency of the dose administration is reduced to safeguard the kidney functions. This is due to accumulation of drugs in the proximal convoluted tubules and decreased clearance. Co-administration of the other drugs that compete with gentamicin through inhibition of megalin ligands and small proteins or administration of antioxidant reduces its toxicity. ${ }^{9}$ Gentamicin induces inflammatory response, activation of cells and cytokine production due to production of ROS; drugs that scavenger these damaging superoxide ions will ultimately protect kidney from adverse effects. ${ }^{1}$

In gentamicin mediated nephrotoxicity; there is significant reduction of renal blood flow and insulin clearance. It was observed that superoxide dismutase has significant increase in both parameters suggesting its protective role. ${ }^{10}$

In our study, there was evident depletion of superoxide dismutase which was more severely in the animals that were not treated with turmeric and relative higher levels of SOD in pre-treated and simultaneously treated with turmeric; this result highlights the antioxidant property of turmeric. Similar potent antioxidant effects of turmeric comparable to Vitamin C, anti-inflammatory property and other protective actions like antimicrobial as well inhibition of platelet aggregation is explored by different researchers. It is also confirmed that turmeric is non-toxic even at higher doses and protective in condition like atherosclerosis, it acts as hepatoprotective and anticarcinogenic. Superoxide dismutase (SOD), Catalyse (CAT) and Glutathione reductase are first line enzymatic defence whose levels are reduced in oxidative stress. In present literature, there is consensus about role of oxidative stress due to reactive oxygen and nitrogen species in gentamicin toxicity. ${ }^{11}$ 
In present study marked damage to renal parenchyma (3.66 \pm 0.24$)$ was observed in animals only treated with gentamicin. We observed that there was spontaneous recovery in renal function parameters to normal and lower grade of renal parenchymal injuries in all animals within one week duration after withdrawal i.e. at $21^{\text {st }}$ day $(1.45 \pm 0.08)$ and maintained thereafter i.e. at $28^{\text {th }}$ day (1.16 \pm 0.08$)$. These results suggest that nephrotoxicity due to gentamicin is reversible after drugs has been discontinued. This extensive damage is due to preferential accumulation of gentamicin in the proximal convoluted tubules which produces variable changes in epithelial cells and graded accordingly. Intracellular changes like hydropic changes with cytoplasmic vacuolation are also seen in some area, similar results i.e. damage and regeneration of epithelium was observed on histopathological examination in kidney after gentamicin administration. Acute renal failure, oxidative stress its scavenging was reported in other studies., ,, $12-14^{-1}$

In our study, oxidative stress was gradually reduced after withdrawal of drug with decreasing grades of parenchymal damage when tested at set duration of interval which was predominant in turmeric treated group. Group which was pre-treated with turmeric for a month shown a lowest oxidative stress, renal tubular damage and maintained kidney function. Statistically significant differences ( $p$ $<0.01$ ) were observed in renal functions but are of clinical irrelevance as they are within normal range.

\section{CONCLUSION}

Gentamicin produces severe but reversible nephrotoxicity at selected doses in albino rats. Underlying oxidative stress evident by decreased levels of SOD whereas turmeric was effective in reducing the oxidative stress in short term treatment but more predominant in long term treated group.

Funding: No funding sources Conflict of interest: None declared

Ethical approval: The study was approved by the Institutional Ethics Committee

\section{REFERENCES}

1. Novoa JML, Quiros Y, Vicent L, Morales AI. New insights into the mechanism of aminoglycosides nephrotoxicity: an integrative point of view. Kidney Interat. 2011;79:33-45.
2. Dontabhakatuni A, Taft DR, Patel M. Gentamicin renal excretion in rats: probing strategies to mitigate drug induced nephrotoxicity. Pharmacol Pharmac. 2016;7:43-55.

3. Padmini MP, Kumar JV. A histopathological study on gentamicin induced nephrotoxicity in experimental albino rats. IOSR. 2012;1:14-7.

4. Irshad M, Chaudhuri PS. Oxidant-antioxidant system: Role and significance in human body. Ind $\mathrm{J}$ Experiment Biol. 2002;40:1223-39.

5. Turmeric-centerchem. V 01-06/05. 40560, 40690, 47740, 47870-1. Available at www.centerchem.com

6. Shukla PK, Khana VK. Protective effect of curcumin against lead neurotoxicity in rat. Human Experiment Toxicol. 2003;22(12):653-58.

7. Houghton DC, Plamp CE, DeFehr JM, Bennett WM. Gentamicin and tobramycin nephrotoxicity. Am J Pathol. 1978;93:137-57.

8. Salgueiro SR, Nunez LG. Animal models mimicking aminoglycoside-induced renal dmage. J Nephro Pharmacol. 2016;5(1):1-3.

9. van der Harst MR, Bull S, Laffont CM, Klein WR. A comparison of in vitro finding with in vivo experiments in equines. Veterinary research Communicat. 2005;29:247-61.

10. Nakajima T, Hashida A. Mechanism for protective effect of free radical scavengers on gentamicin induced nephropathy in rats. Am J.Physiol. 1994;266:425-31.

11. Akram M, Shahab-uddin, Ahemed A, Khan U. Curcuma longa and curcumin: A review article. Rom J Biol. 2010;55(2):65-70.

12. Qadir MI, Tahir M, Lone KP, Munir B. Protective role ginseng against Gentamicin induced changes in albino mice. J Ayub Med Coll Abbottabad. 2011;23(4):53-7.

13. Shalaby MA. Hammoda AA. Evaluation of nephroprotective and diuretic effects of parsley and turmeric herbs on gentamicin nephrotoxic rats. WJPPS. 2014;3(12):1729-44.

14. Azab AES, Fetouh FA, Albasha MO. Nephroprotective effects of curcumin, rosemary and propolis against gentamicin induced toxicity in guinea pigs: Morphological and biochemical study. Am J Clinical Experiment med. 2014;2(2):28-35.

Cite this article as: Jogdand SD, Shinde R, Sinha V, Chandrakar N. Nephroprotective effect of turmeric on oxidative stress, renal histopathology and toxicity induced by gentamicin. Int J Basic Clin Pharmacol 2017;6:1282-6. 